\title{
Evolução favorável de recidiva de infecção por varicela zoster (cobreiro) durante uso de natalizumabe em paciente com esclerose múltipla: relato de caso
}

\section{Favorable outcome of shingles during natalizumab treatment in a patient with multiple sclerosis: case report}

Diego Rafhael Soares Carvalho Feitosa ${ }^{1}$. Francisco de Assis Aquino Gondim² ${ }^{2}$ Galeno Vieira Rochaํ.

1 Residência em Neurologia, Hospital Universitário Walter Cantídio (HUWC), Fortaleza, Ceará, Brasil. 2 Doutor em Farmacologia pela Universidade Federal do Ceará (UFC). Docente do Departamento de Medicina Clínica, Serviço de Neurologia, Universidade Federal do Ceará, Fortaleza, Ceará, Brasil.

\section{RESUMO}

Objetivo: relatar o caso de recidiva de herpes-zoster (HZ) em paciente com esclerose múltipla (EM) em uso de tratamento imunomodulador (natalizumabe) com evolução favorável. Descrição de caso: mulher de 39 anos com diagnóstico de EM há 1 ano e 6 meses, previamente tratada com interferon 1a, começou tratamento com natalizumabe, desenvolvendo HZ após três meses de tratamento. HZ foi tratado com aciclovir, por sete dias, via oral. Seguimento ambulatorial após três meses de tratamento mostrou resolução completa das lesões, sem desencadeamento de neuralgia pós-herpética. Conclusões: As novas terapias para a EM podem estar relacionadas a diferentes tipos de efeitos adversos. Nem todos os casos de HZ, associados com novas terapias para EM, evoluem de forma desfavorável. Estudos são necessários para reconhecer os fatores de riscos para as formas graves de $\mathrm{HZ}$ em tais pacientes.

Palavras-chave: Esclerose múltipla. Natalizumabe. Herpes Zoster.

\section{ABSTRACT}

Objective: To report a case of a benign (favorable) course of shingles in a patient with multiple sclerosis (MS) treated with immunomodulatory treatment (natalizumab). Case description: A 39 year-old woman diagnosed with MS for one year and a half, previously treated with interferon 1a, began treatment with natalizumab, developing HZ after 3 months of treatment. Shingles was treated with p.o. acyclovir for 7 days. Outpatient follow-up revealed complete resolution of the skin lesions, without the development of post- herpetic neuralgia. Conclusions: The new therapies for MS may be associated with different types of adverse effects. Not all cases of shingles associated with new therapies for MS progress in an unfavorable fashion. Studies are necessary to recognize the risk factors for the severe forms of shingles in this group of patients.

Keywords: Multiple sclerosis. Natalizumabe. Herpes Zoster.

Autor correspondente: Francisco de Assis Aquino Gondim, Rua Professor Costa Mendes, 1608, Departamento de Medicina Clínica, Rodolfo Teofilo, Fortaleza, Ceará. CEP: 60430-140.Telefone: +55 85 3366-8354/8333. E-mail: gondimfranc@gmail.com

Conflito de interesses: Não há qualquer conflito de interesses por parte de qualquer um dos autores.

Recebido em: 02 Jan 2016; Revisado em: 01 Ago 2016; Aceito em: 09 Ago 2016. 


\section{INTRODUÇÃO}

A esclerose múltipla (EM) é uma doença desmielinizante do sistema nervoso central, associada a presença de inflamação, gliose, perda e disfunção neuronal. O início ocorre geralmente entre 20 e 40 anos de idade, mais comum em mulheres (duas a três vezes). ${ }^{1}$ É a segunda causa de incapacidade neurológica, superada pelo traumatismo craniano. Apesar das variáveis apresentações da EM, são identificados pelo menos dois tipos de cursos clínicos. O mais comum no adulto jovem, chamado de recorrente/remitente, cursa com déficits neurológicos transitórios, apresentando imprevisibilidade quanto ao tempo de instalação e às características do próximo surto. Destacam-se, também, as formas de curso progressivo, onde os déficits neurológicos instalados são intensificados, sem remissão, com comprometimento dos sistema piramidal e/ou cerebelar e concomitante incapacidade motora.

Desde o início do século XXI, novas terapias, associadas com imunossupressão ou imunomodulação, vêm substituindo as terapias anteriores, associando-se com melhores prognósticos. Dentre as novas terapias, destaca-se o anticorpo recombinante humanizado da anti- $\alpha$-4-integrina, chamado de natalizumabe. ${ }^{2}$ Apesar de conferir uma maior probabilidade de controle da doença, com diminuição da progressão e do número de recidivas, também pode estar associado com novos efeitos colaterais importantes, destacando-se a leucoencefalopatia multifocal progressiva (LEMP) ${ }^{3}$ e as infecções recidivantes por varicela-zoster (cobreiro). ${ }^{4}$

Anteriormente, relatamos um paciente com recidiva de $\mathrm{HZ}$ associada com o uso de fingolimode, também com prognóstico favorável..$^{5}$ Entretanto, até o presente, não existem critérios específicos para avaliar o risco de desenvolvimento de formas graves de $\mathrm{HZ}$ em pacientes que estão em uso de novas terapias para EM. O presente trabalho descreve o curso clínico brando de infecção por herpes zóster redicivante em uma paciente com EM tratada com natalizumabe.

\section{RELATO DE CASO}

Paciente feminina, 39 anos, natural de Iracema-Ceará, recebeu o diagnóstico de EM em sua forma recorrente/remitente em 2014, satisfazendo os critérios de McDonald ${ }^{6}$ após quadro de paralisia facial a direita, parestesias e hemiparesia em dimidio esquerdo. Evoluiu com novo episódio de fraqueza, perda da sensibilidade tátil em membros inferiores e superiores e hiper-reflexia. As imagens de ressonância nuclear magnética (RNM) de encéfalo apresentavam sinais de desmielinização com formas e topografias compatíveis com EM. Havia relato de varicela na infância, sem demonstração laboratorial de infecção prévia. O primeiro curso de tratamento para EM, de julho de 2014 a julho de 2015, foi realizado com interferon beta-1a. Por falha terapêutica, em agosto de 2015, o tratamento foi substituído por natalizumabe mensalmente.

Após três infusões, a paciente apresentou clínica de prurido, eritema e dor localizada na região de dermátomo torácico ao nível de T6-T7 (Figuras 1 e 2), sem novas alterações ao exame neurológico. No segundo dia de evolução, surgiram erupções máculo-vesiculares compatíveis com varicela zóster. O natalizumabe foi descontinuado e foi iniciado uso de aciclovir, por via oral, durante sete dias ( $800 \mathrm{mg} /$ dia). Após uma semana, as lesões melhoraram sem dor neuropática residual. O quadro neurológico e funcional não apresentou alterações.

Figura 1. Infecção por herpes-zóster (cobreiro) em dermátomo T-6 e T-7.

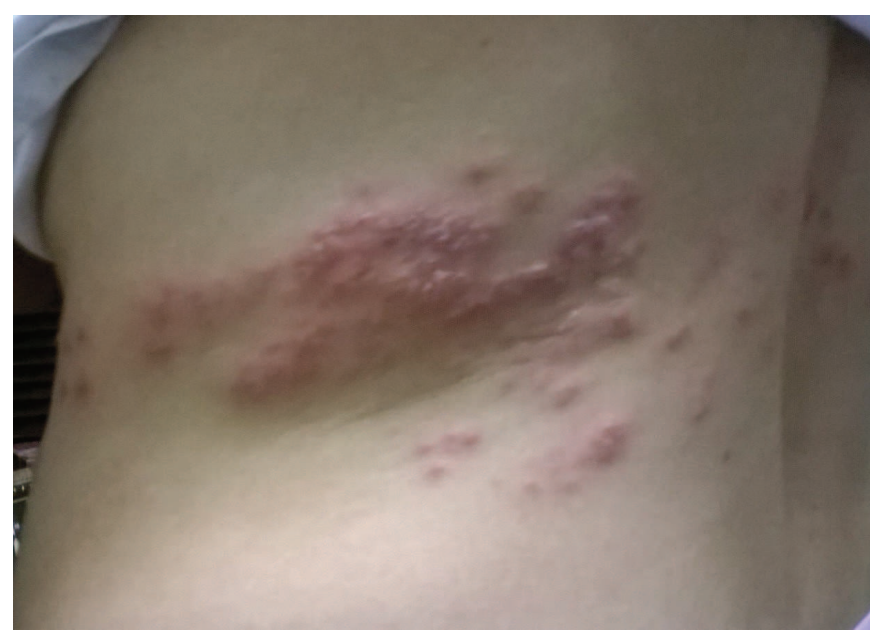

Figura 2. Lesões eritemato-vesiculares caracterizando herpes-zóster.

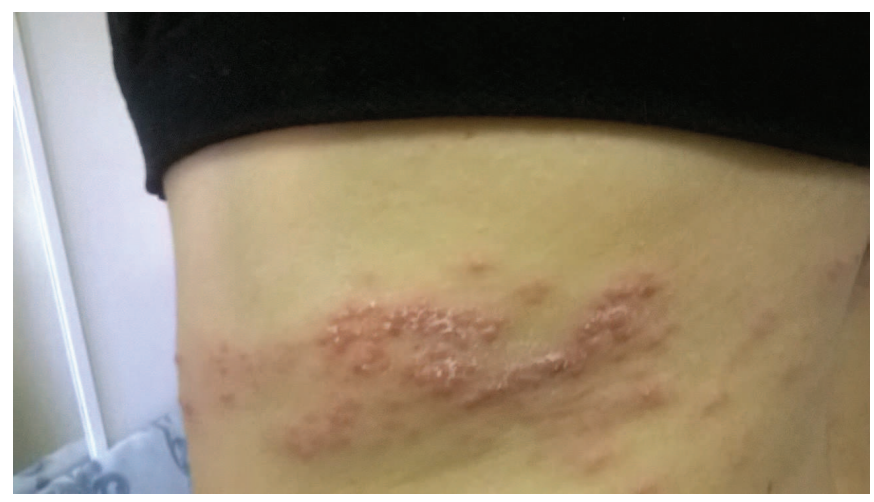

Após três meses, a paciente não evidenciou lesões dermatológicas em atividade ou sintomas clínicos de envolvimento neurológico. Foi iniciado o uso de acetato de glatiramer $20 \mathrm{mg} /$ dia para dar continuidade ao tratamento de EM.

\section{DISCUSSÃO}

O Vírus Varicela Zóster (VZV) permanece em estado de latência nos gânglios da raiz dorsal, após infecção primária. Condições clínicas que desencadeiem queda das respostas imunes celulares específicas ao VZV podem ocasionar quadros de HZ. Reativações conhecidas, na cultura popular, com o nome de cobreiro e caracterizadas pelo aparecimento de lesões na pele, com manchas avermelhadas que evoluem para vesículas ou pequenas bolhas com líquido claro no seu interior, situadas em trajetos de nervos sensitivos, são muitas 
vezes relacionadas com dor neuropática. A imunossupressão causada pelas novas terapias para EM e o desfecho associado ao aumento do risco de infecção por VZV, apresentam importância clínica, principalmente em pacientes em uso do natalizumabe, agente que tem ação dirigida à $\alpha$-1-integrina na barreira hematoencefálica, impedindo que as células T saiam da circulação e entrem no Sistema Nervoso Central (SNC), reduzindo assim os ataques à mielina. ${ }^{7}$

Em ensaios clínicos, as infecções por vírus do herpes (VZV, bem como pelo vírus Herpes simplex) foram ligeiramente mais frequentes em pacientes tratados com natalizumabe do que nos doentes tratados com placebo. ${ }^{8}$ Muitas publicações ressaltam a real possibilidade de pacientes tratados com natalizumabe desenvolverem quadros clínicos e neurológicos graves, expressos por esofagite, retinite, encefalite herpética e vasculite secundária à VZV. ${ }^{9}$ Descrevem-se, também, em pacientes tratados para EM com natalizumabe, quadros de mielite aguda por VZV, além dos tipicamente brandos, caracterizados por acomentimento dermatológico e sensitivo. Diante dessas informações, tornou-se recomendável a vacinação contra VZV nos pacientes soronegativos para indicação do natalizumabe e do fingolimode. Apesar disso, não existem estudos sistemáticos sobre os riscos e benefícios da prescrição de medicamentos anti-herpéticos profiláticos ou vacinas, considerando que as decisões terapêuticas devem ser

\section{REFERÊNCIAS}

1. Voskuhl RR, Gold SM. Sex-related factors in multiple sclerosis: genetic, hormonal and environmental contributions. Nat Rev Neurol. 2012;8(5):255-63.

2. Polman $\mathrm{CH}$, O'Connor PW, Havrdova E, Hutchinson $\mathrm{M}$, Kappos L, Miller DH, et al. A randomized, placebo-controlled trial of natalizumab for relapsing multiple sclerosis. N Engl J Med. 2006;354(9):899-910.

3. Bloomgren G, Richman S, Hotermans C, Subramanyam M, Goelz $\mathrm{S}$, Natarajan A, et al. Risk of natalizumab-associated progressive multifocal leukoencephalopathy. N Engl J Med. 2012;366(20):1870-80.

4. Fragoso YD, Brooks JB, Gomes S, Oliveira FT, Gama PD. Report of three cases of herpes zoster during treatment with natalizumab. CNS Neurosci Ther. 2013;19(4):280-1.

5. Morais AA, Tavares JW Jr, Melo AP Neto, Lima FP, Teixeira CA, Gondim FA. Evolução branda de recidiva de infecção por varicela zoster após tratamento com fingolimode em paciente com esclerose múltipla: relato de caso. J Health Biol Sc. 2016;4(1):38-40.

6. Polman CH, Reingold SC, Banwell B, Clanet M, Cohen JA, Filippi M, et al. Diagnostic criteria for multiple sclerosis: 2010 revisions to the McDonald criteria. Ann Neurol. 2011;69(2):292-302. individualizadas. Sem imunização prévia, a literatura mostra um bom desfecho clínico, em casos de HZ ocorrendo em pacientes com EM tratados com natalizumabe, realizando-se suspensão imediata da droga em questão e da instituição do tratamento anti-retroviral específico. ${ }^{10}$

Propostas de profilaxia antiviral contra herpes vírus, em pacientes tratados com alemtuzumabe (outro fármaco com ação imunomoduladora), tem sido preconizada nos primeiros 30 dias de terapêutica com tal medicação. O protocolo inclui a administração de aciclovir $200 \mathrm{mg}$, duas vezes ao dia, desde o primeiro dia de infusão do alemtuzumabe até pelo menos 1 (um) mês após o término desta medicação. ${ }^{11}$

O caso em questão mostrou evolução branda do HZ, sem piora no padrão neurológico de base ou aparecimento de dor neuropática, após a suspensão do natalizumabe e início de terapia anti-retroviral específica (aciclovir) de forma precoce. O relato tem como importância o fato de destacar a ocorrência de HZ associado ao uso das novas terapêuticas imunomoduladoras em relação a EM, enfatizando a necessidade do conhecimento da patologia, dos sinais cutâneos, da significância do diagnóstico precoce e da instituição de terapêutica específica ao caso. Dessa forma, as complicações, como dor neuropática, podem ser minimizadas ou até mesmo evitadas.

7. European Medicines Agency. Tysabri: natalizumab [Internet]. Londres: European Medicines Agency; 2014[acesso 2016 Maio 25]. Disponível em: http://www.ema.europa.eu/ema/ index. jsp? curl=pages/medicines/human/medicines/000603/human med_001119.jsp\&mid=WC0b01ac058001d124

8. Schiess NZ, Hayward G, Calabresi P, Nath A. Reactivation of herpes viruses in multiple sclerosis patients on natalizumab therapy [Internet]. Seattle, (WA): American Academy of Neurology's; 2009. [acesso 2016 Maio 25]. Disponível em: https://www.aan.com/Press Room/home/PressRelease/687

9. Yeung J, Cauquil C, Saliou G, Nasser G, Rostomashvili S, Adams $\mathrm{D}$, et al. Varicella-zoster virus acute myelitis in a patient with multiple sclerosis treated with natalizumab. Neurology. 2013;80(19):1812-13.

10. Robles L, Prieto P, Hutton GJ, Rivera VM. Occurrence of Herpes Zoster in Multiple Sclerosis Patients Treated with Natalizumab. International Journal of MS Care. 2009;11(Suppl 2):S:43.

11. Havrdova E, Horakova D, Kovarova I. Alemtuzumab in the treatment of multiple sclerosis: key clinical trial results and considerations for use. Ther Adv Neurol Disord. 2015;8(1):31-45.

\section{Como citar:}

Feitosa DR, Gondim FA, Rocha GV. Evolução favorável de recidiva de infecção por varicela zoster (cobreiro) durante uso de natalizumabe em paciente com esclerose múltipla: relato de caso. 2017 jan-abr;57(1):66-68. 